\title{
Spastic quadraparesis following a relatively minor injury - the 'os odontoideum'
}

\author{
S Moosa, MB ChB, BSc Hons (Pharm), \\ MPhil, FC Rad (Diag) \\ Department of Radiology, Groote Schuur Hospital and \\ University of Cape Town \\ S Andronikou, MB BCh, FC Rad (Diag), \\ FRCR (Lond), PhD \\ Department of Radiology, Tygerberg Hospital and \\ Stellenbosch University
}

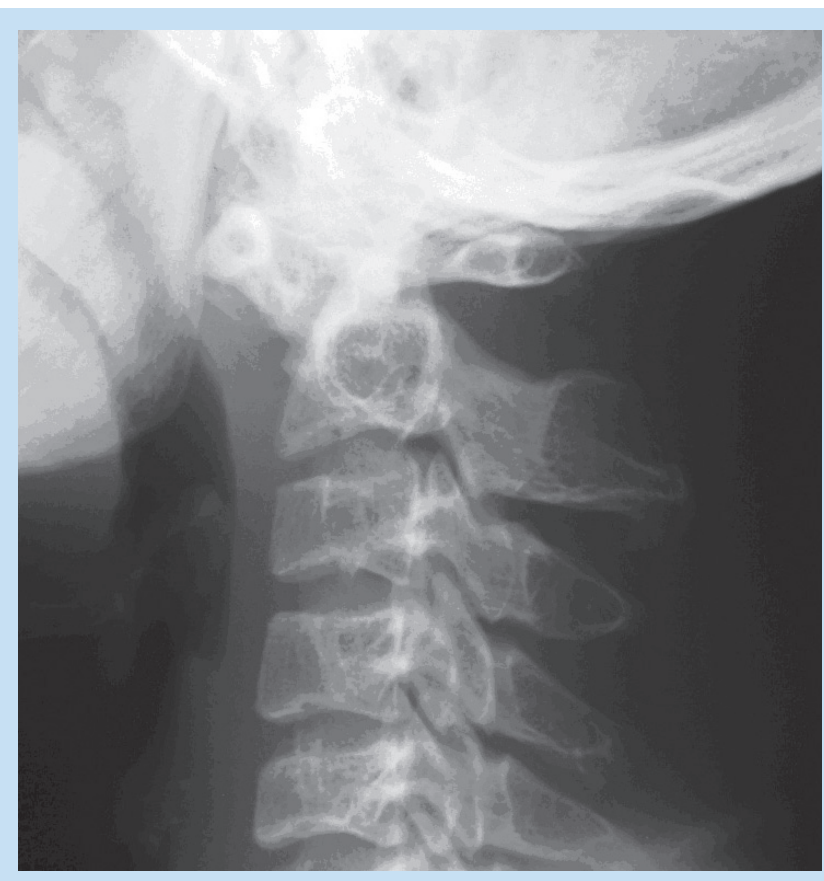

Fig. 1. Lateral neck view demonstrating an atlantodental interval (ADI) of 11 $\mathrm{mm}$ compared to the normal for adult males of up to $3 \mathrm{~mm}$. Well-corticated bony ossicles are visualised superior and inferior to the anterior arch. The dens is small and the posterior arch of $\mathrm{C} 1$ is displaced anteriorly. The spinal canal is reduced in diameter.

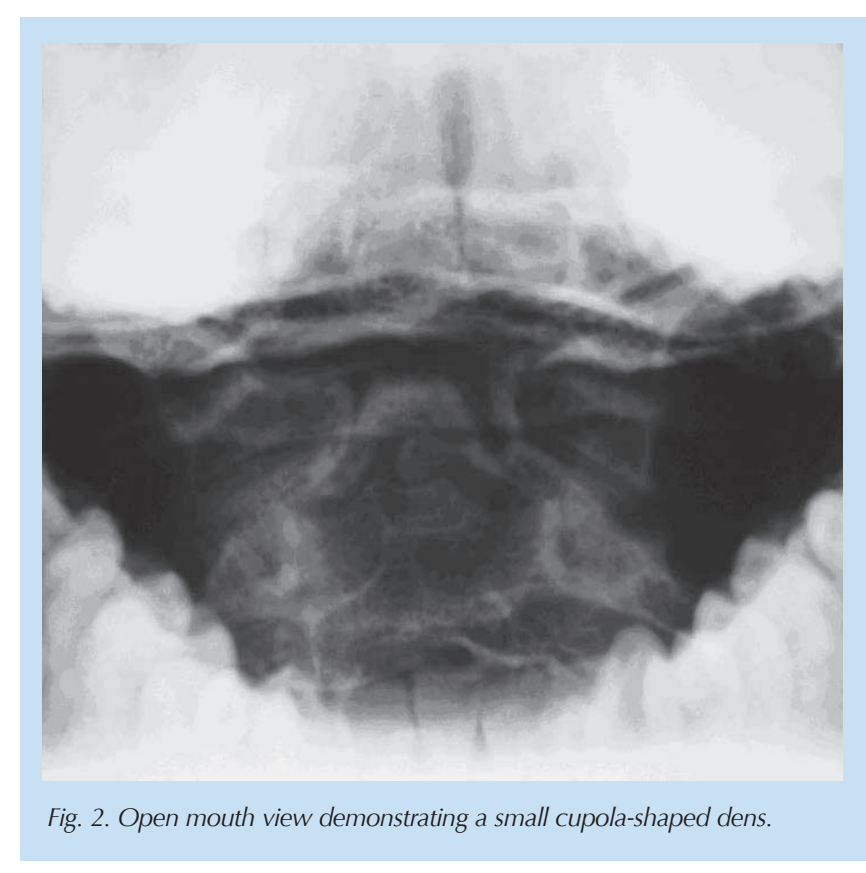

He was admitted with a diagnosis of spastic quadraparesis. He has recovered to some degree, being able to 


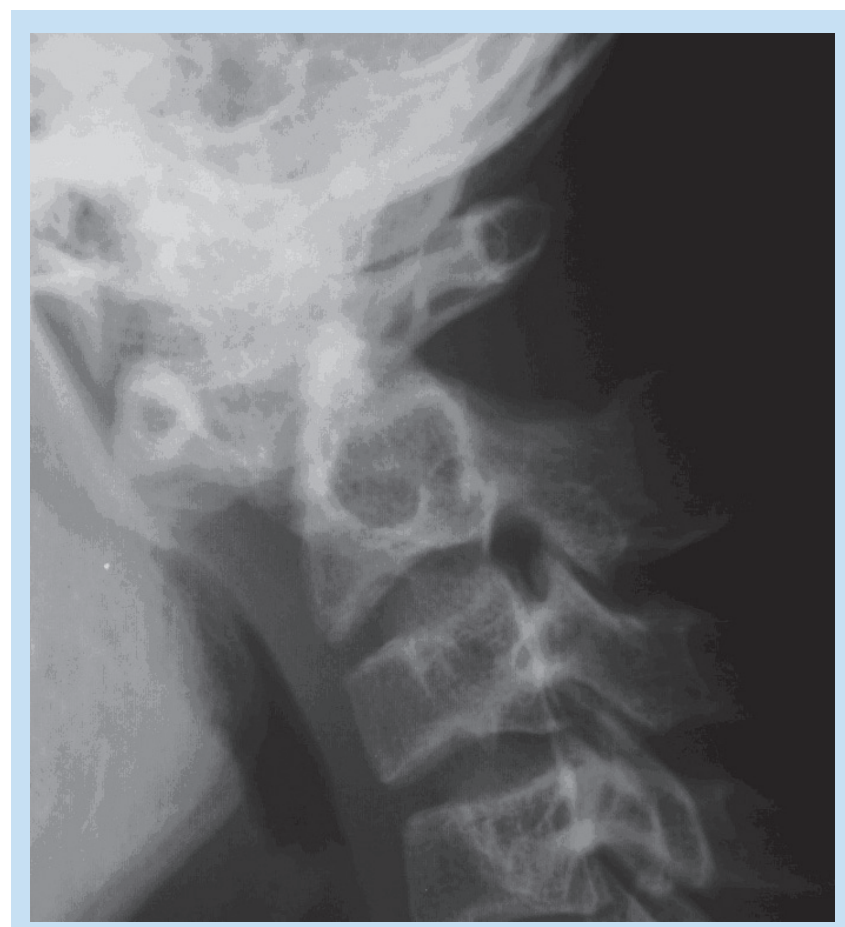

Fig. 3. Flexion view demonstrating a decrease in spinal canal diameter with flexion while the $A D I$ increases in dimensions.

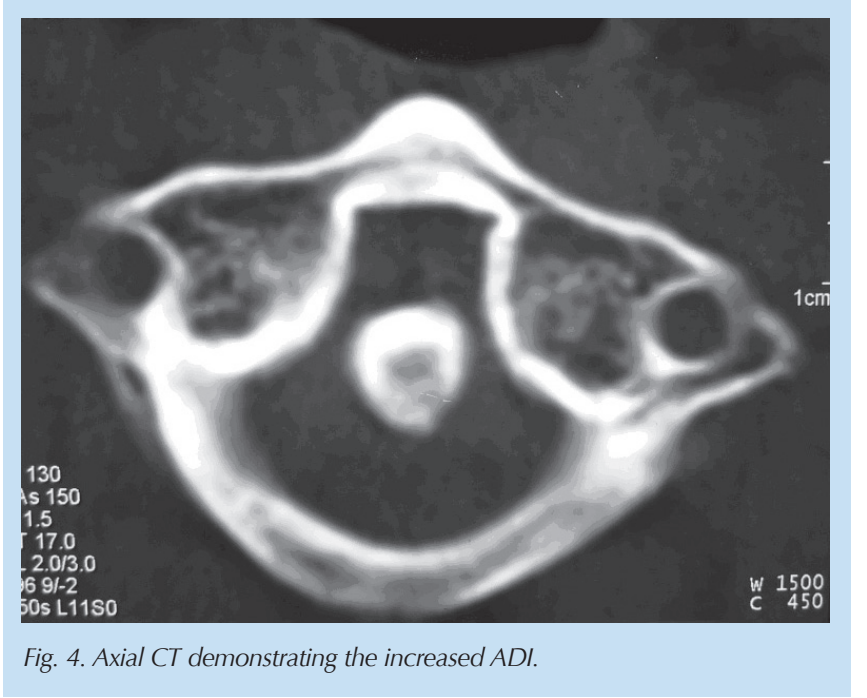

walk unaided for short distances and has regained some power in both hands. Plain film, computed tomography (CT) scan and magnetic resonance (MR) images are presented in Figs 1 - 5. A diagnosis of os odontoideum with C1-2 subluxation and high cervical spinal cord compression was made.

\section{Discussion}

Os odontoideum (Greek 'tooth like') is a rare anomaly of the second cervical vertebra which was first

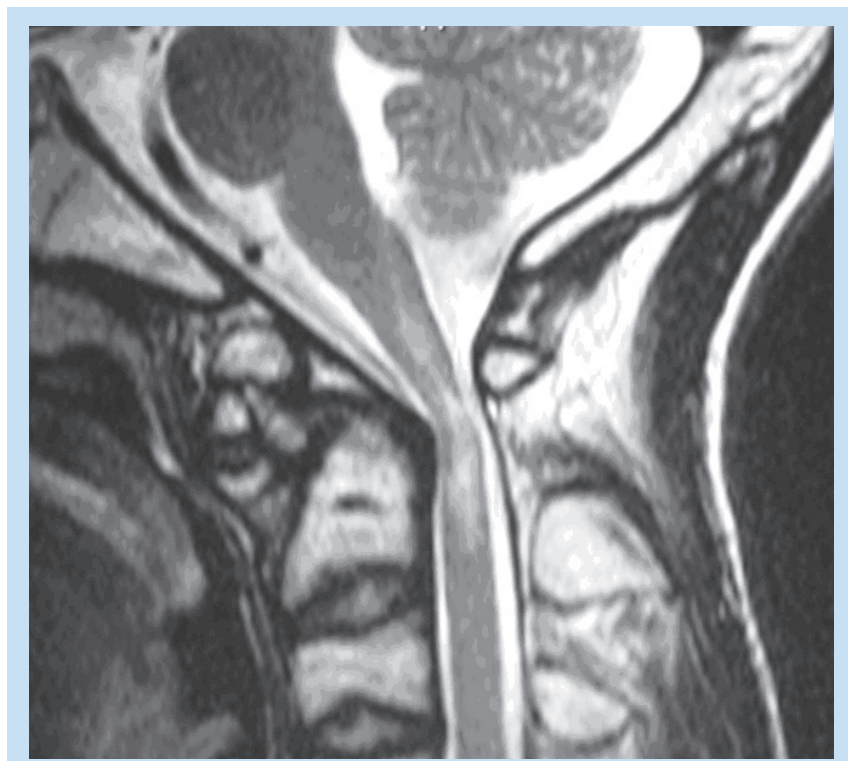

Fig. 5. Sagittal T2-weighted MR image demonstrating abnormal high signal in the cord which is kinked at the upper cervical level. The markedly narrowed spinal canal and anterior CSF effacement is well visualised.

described from postmortem studies by Giacomini in 1886. ${ }^{1}$ By definition, an os odontoideum is a small accessory ossicle with smooth, well-corticated borders that is separated from the base of a shortened odontoid process. The size and shape of the ossicle can vary. The atlanto-axial joint exhibits the greatest mobility in the vertebral column and is also the least stable. ${ }^{1}$ Os odontoideum frequently leads to atlanto-axial instability with spinal-cord compression. ${ }^{2}$ Atlanto-axial instability can arise from multiple causes including inflammatory arthritides, trauma, infection, laxity of the transverse ligament, e.g. trisomy 21 and other congenital abnormalities. Plain cervical spinal radiographs are usually sufficient to obtain a diagnosis.

Os odontoideum has been classified into two anatomic types, orthotopic and dystopic. Orthotopic describes an ossicle that moves with the anterior arch of $\mathrm{C} 1$, whereas dystopic defines an ossicle that is functionally fused to the basion. Minor trauma can produce symptoms when the dystopic os is freed and subluxes anterior to the arch of $\mathrm{C} 1 .^{3}$

The gap between the os odontoideum and the odontoid process extends to above the superior articular facet of the atlanto-axial joint. This is in contrast to an acute fracture or non-union of the odontoid in which the gap is narrow and irregular and extends into the body of the axis below the level of the superior facet.' 
Os odontoideum is associated with hypoplasia of the posterior arch as well as anterior arch hypertrophy, compensating for the increased stress placed on the latter. It occurs commonly in trisomy 21, Klippel-Feil and other craniocervical malformations.

The aetiology of os odontoideum remains unsettled but this does not play a major role in its diagnosis or management. ${ }^{3}$ The proponents who feel that it is a congenital abnormality suggest that the mechanism involves the failure of the os terminale to fuse, or failure of the main part of the odontoid process to fuse with the body of the axis. ${ }^{1}$ (SA: OK?)

Fielding et al. ${ }^{4}$ attributed os odontoideum to a disunited fracture by presenting cases with a history of trauma and previously normal radiographs. They propose that subsequent to an odontoid fracture, the proximal portion is pulled by the contraction of the alar ligament and non-union is facilitated by vascular interruption. In our case, a second ossicle inferior to the anterior arch is uncommon and probably points to a traumatic origin.

The literature supports the ability of cervical plain films to establish the diagnosis of os odontoideum but their sensitivity and specificity as well as the need for additional confirmatory studies is still unreported. ${ }^{3}$ Flexion and extension plain films have been used to depict the degree of $\mathrm{C} 1$ and $\mathrm{C} 2$ instability. Anterior subluxation of the os in relation to the body of $\mathrm{C} 2$, as was the case in this patient, predominates over posterior subluxation. ${ }^{3}$ CT scans have been used to better define the bony anatomy of the os odontoideum and the odontoid process. The role of MRI has been to evaluate the spinal cord and to provide additional anatomical information. Both CT and MRI assist in operative planning of unstable os odontoideum.

The degree of C1-2 instability does not seem to correlate with the neurological status in patients with os odontoideum and a sagittal spinal canal diameter on plain films of $13 \mathrm{~mm}$ or less is strongly associated with myelopathy. ${ }^{3}$ Asymptomatic patients with os odontoideum, with or without C1-2 instability may be managed with clinical and radiological surveillance. Those with symptoms and/or neurological signs and C1-2 instability may be managed with posterior C1-2 internal fixation and fusion. ${ }^{3}$

\section{Conclusion}

Os odontoideum is a potentially mechanically unstable condition which is associated with trisomy 21 and other congenital conditions. Minor trauma may result in severe spinal cord injury. Cervical plain films are usually diagnostic. Both CT and MRI assist in operative planning. The incidental detection of an os odontoideum should not be considered as a variant anomaly but should be referred for appropriate spinal evaluation and surveillance. This may have special relevance in children whose sporting activities may have to be restricted.

\footnotetext{
1. Forlin E, Herscovici D, Bowden JR. Understanding the os odontoideum. Orthopaedic Review 1992; 21 1441-1446.

2. Dai L, Wen Y, Bin N, Jia I. Os odontoideum: etiology, diagnosis and management. Surg Neurol 2000; 53: 106-109.

3. Os odontoideum. Neurosurgery 2002; 50: S148-S155.

4. Fielding JW, Hensinger RN, Hawkins RJ. Os odontoideum. J Bone Joint Surg Am 1980; 62: 376-383.
} 